\title{
Análise estratégica de um centro de genética médica em um instituto de pesquisa nacional em saúde no Brasil: desafios para o SUS
}

\author{
Strategic analysis of a medical genetics center at a national health \\ research institute in Brazil: challenges for the country's public \\ health system
}

Elizabeth Artmann (https://orcid.org/0000-0002-8690-5964) ${ }^{1}$

Juan Clinton Llerena Junior (https://orcid.org/0000-0002-4308-3841) ${ }^{2}$

Laura Terezina Pereira (https://orcid.org/0000-0003-2239-3821) ${ }^{2}$

Luciane Binsfeld (https://orcid.org/0000-0001-7513-5266) ${ }^{2}$

Francisco Javier Uribe Rivera (https://orcid.org/0000-0002-3177-5170) ${ }^{1}$

${ }^{1}$ Escola Nacional de Saúde Pública Sergio Arouca, Fundação Oswaldo Cruz. Leopoldo Bulhões 1.480, sala 710 , Manguinhos. 21040-360 Rio de Janeiro RJ Brasil.

bethartmann@gmail.com

${ }^{2}$ Instituto Fernandes

Figueira. Fundação Oswaldo

Cruz. Rio de Janeiro RJ

Brasil.

\begin{abstract}
Objective: to discuss strategic challenges in complex health organizations sustained by an analysis in a Medical Genetics Center at a National Health Research Institute in Brazil. Method: Démarche Stratégique adapted for use with research institutes, including the policy formulation function. The study used institution's databases and workshops with key actors between Dec/2014 and Jun/2015. Results: Were defined the segments: clinical diagnosis, $l y$ sosomal diseases, osteogenesis imperfecta, genetic counseling, cytogenetics, biochemistry, and genom$i c s$. The findings reveal that the segments are aligned with the Institute's mission when it comes to referral care, although investment is required in strategic areas. The clinical diagnosis, lysosomal diseases, and genomics segments stood out in the scientific output portfolio. The findings show that progress needs to be made with policy implementation and planning to improve access to new technologies, together with investment in training and development, and the strengthening of coordination and collaboration between research institutes and referral services and between the wider healthcare network and research, care, and teaching. The inclusion of the policy formulation dimension was essential to highlight the unique role medical genetics plays in Brazil's public health system and the importance of developing action plans in the field of public health.
\end{abstract}

Key words Medical genetics, Health planning, Prospective analysis, Hospital management, Health policy
Resumo $O$ objetivo deste artigo é discutir os desafios estratégicos em organizações complexas de saúde apoiado na análise da posição estratégica em um centro de genética médica em um instituto nacional de pesquisa em saúde brasileiro. Utilizou-se a démarche stratégique adaptada para institutos de pesquisa, incorporando a função formulação de politicas com apoio de bancos de dados institucionais, livros de registros, reuniões com atores-chave e oficinas de trabalho de dezembro de 2014 a junho de 2015. Foram definidos os segmentos diagnóstico clínico, doenças lisossômicas, osteogênese imperfeita, aconselhamento genético, citogenética, bioquímica e genômica. Estes mostraram-se adequados à missão institucional no que se refere à atenção de referência, necessitando investimentos em pontos estratégicos. Na produção científica destacaram-se os segmentos diagnóstico clínico, doenças lisossômicas e genômica. Os resultados mostraram que é preciso avançar na implementação de políticas e no planejamento para ampliar o acesso da população às novas tecnologias, investindo em formação e na articulação assistência/pesquisa/ensino. A incorporação da dimensão formulação de política foi fundamental para indicar o papel diferencial da genética médica no SUS.

Palavras-chave Genética médica, Planejamento em saúde, Análise prospectiva, Gestão hospitalar, Política de saúde 


\section{Introdução}

Segundo a Organização Mundial da Saúde, as doenças genéticas atingem de $3 \%$ a $10 \%$ da população mundial. Tais enfermidades são definidas como agravos à saúde decorrentes da alteração do material genético (DNA) e, na maioria das vezes, estão presentes desde a concepção. Manifestam-se de formas distintas e em diferentes períodos da vida, podendo ocorrer desde a gestação, como no caso dos defeitos congênitos, até a vida adulta, como no caso de um câncer hereditário ${ }^{1-3}$.

No Brasil, dados epidemiológicos demonstram que os defeitos congênitos, mais especificamente as malformações congênitas, são a segunda causa de mortalidade infantil precoce, seguindo a tendência mundial ${ }^{4}$. O câncer representa a segunda causa de óbito na população geral e, desse total, estima-se que entre 5\% e 10\% sejam hereditários, configurando síndromes de predisposição para o câncer herdadas em sua grande maioria de forma autossômica dominante $e^{5-7}$. Tais doenças, além de um impacto desfavorável ao indivíduo afetado, geram grande impacto social no contexto das famílias e da sociedade. As enfermidades genéticas não podem ser negligenciadas, especialmente pela grande carga que representam no tempo de vida sã perdida (DALY - disability adjusted life year). O importante avanço da genética no nível molecular, que permitiu melhor compreensão e conhecimento das doenças, amplia o potencial de contribuição dessa área na atenção em saúde dos grupos populacionais afetados. ${ }^{2,3}$

O reconhecimento da relevância do tema levou à inclusão da genética médica no Sistema Único de Saúde (SUS), e a discussão sobre o diagnóstico, tratamento e organização do cuidado às doenças genéticas resultou na publicação da Política Nacional de Atenção Integral em Genética Clínica ${ }^{8}$ e na Política Nacional de Atenção Integral ao Indivíduo com uma Doença Rara?.

Contudo, vários problemas ainda precisam ser superados no caso da atenção à genética no Brasil, como a quantidade inadequada de consultas especializadas disponíveis, a centralização dos serviços em grandes centros urbanos, as dificuldades de acesso aos serviços especializados e a articulação entre os níveis de atenção. As questões elencadas são fatores que dificultam o acesso e contribuem para a desassistência de famílias e pessoas com doenças genéticas e para as deficiências no cuidado às suas necessidades de saúde ${ }^{2,5}$.

Com o objetivo de enfrentar os desafios dessa área, foi criado em 1989 o Centro de Genética Médica (CGM) no Instituto Fernandes
Figueira (IFF). Este vem atuando com a missão de desenvolver atividades relacionadas à atenção de referência, pesquisa e ensino no município e no estado do Rio de Janeiro, assim como no apoio à formulação de políticas públicas para questões envolvendo a genética médica junto ao Ministério da Saúde. Em 2016, o CGM do IFF se credenciou como centro de referência para atendimento integral ao indivíduo com uma doença rara (Centro de Doenças Raras), atendendo aos critérios demandados pelo Ministério da Saúde ${ }^{10}$.

O IFF é uma unidade da Fundação Oswaldo Cruz (Fiocruz) e se reconfigurou em Instituto Nacional de Saúde da Mulher, da Criança e do Adolescente, por meio da Portaria no 4.159, de 21 de dezembro de $2010^{11}$. A partir dessa mudança, sua atribuição/missão passa a se caracterizar por uma ação mais intensa de assessoria estratégica na proposição, implementação e avaliação de políticas e programas que promovam a ampliação do acesso, o desenvolvimento de modelos de atenção, a pesquisa e inovação tecnológica e a atuação como apoio à coordenação de redes de atenção à saúde da mulher, da criança e do adolescente ${ }^{11}$.

Essa reconfiguração vem exigindo mudanças estratégicas em suas unidades/departamentos, incluindo o CGM, relacionadas à necessidade de fortalecimento de suas competências para ajuste da organização à nova missão. Para tanto, o IFF vem promovendo debates em torno da redefinição de seu perfil assistencial e seu modelo de gestão, que se baseiam nos princípios e diretrizes da Política Nacional de Humanização $(\mathrm{PNH})^{12-14}$ e da gestão estratégica hospitalar, utilizando como referencial teórico-metodológico a démarche stratégique (DS) ${ }^{15,16}$.

Esse referencial propõe a redefinição da missão de hospitais e organizações complexas de saúde e utiliza o critério de oportunidade visando favorecer a integração do hospital em uma rede de cuidados estruturada com eficácia e eficiência, a partir de um processo de transformação cultural progressiva ${ }^{15}$.

Essa perspectiva se apoia em elementos da microeconomia, do campo da estratégia e da política e da área do desenvolvimento organizacional e cultural, e introduz também algumas categorias próprias da área de saúde e da epidemiologia, reconhecendo a especificidade do setor público de saúde. Esse enfoque teórico-metodológico, desenhado originalmente por autores franceses, tem sido adaptado à realidade brasilei$\mathrm{ra}^{17,18}$ e aplicado em unidades hospitalares e em institutos de pesquisa ${ }^{19}$. 
Essa abordagem aponta também para a responsabilidade dos atores no desenvolvimento de processos comunicativos e de negociação internos e externos que redundem em acordos relativos aos projetos assistenciais pertinentes a uma rede de serviços, assim como na constituição de espaços colegiados que agreguem trabalhadores, gestores e usuários em torno de processos decisórios mais participativos e democráticos ${ }^{16}$.

Nesse sentido, tanto os dispositivos da $\mathrm{PNH}$ como as premissas do enfoque DS encontram sinergia, pois se opõem a uma definição normativa e tecnocrática de diretrizes e buscam um lugar de comunicação que promova um elo entre a gerência estratégica e os centros assistenciais, para que os atores possam definir estratégias e construir um projeto comum.

Considerando o cenário de reconfiguração da missão do IFF em instituto nacional e os desafios estratégicos em organizações complexas de saúde ${ }^{20}$ o objetivo deste trabalho foi discutir a posição estratégica do CGM e ações que potencializem suas atividades de atenção de referência, ensino e pesquisa. A singularidade do presente estudo se deve à realização da análise a partir da aplicação da démarche stratégique, incorporando de forma inédita a função de apoio à formulação de políticas.

\section{Procedimentos metodológicos}

A área de estudo selecionada foi a genética médica, por sua posição estratégica na rede de atenção, pela relevância que as doenças genéticas assumem no contexto nacional e pelo potencial de inovação tecnológica na área de pesquisa clínica e cuidado em saúde ${ }^{21}$.

O CGM/IFF dispõe de diversos serviços de referência, como: laboratórios de citogenética clínica; genética molecular aplicada; centro de infusões venosas e terapia de reposição enzimática voltados para as doenças metabólicas; diagnóstico dos erros inatos do metabolismo; ambulatórios em genética médica geral (defeitos congênitos, síndromes genéticas, doenças do desenvolvimento); ambulatórios de osteogênese imperfeita, erros inatos do metabolismo, prénatal de alto risco fetal e aconselhamento genético. São oferecidas também ações de formação de profissionais em pós-graduação lato e stricto sensu para médicos geneticistas, coordenação de linhas e coortes de pesquisa em genômica e o desenvolvimento e participação em ensaios clínicos nacionais.
No âmbito do apoio à formulação de políticas públicas de saúde, o CGM participa do desenvolvimento de diretrizes clínicas, presta consultoria às áreas técnicas do Ministério da Saúde, coordena o Centro de Referência para o Tratamento da Osteogênese Imperfeita (CROIFF), capacita e orienta profissionais no manejo das doenças genéticas e tem parceria com o Centro Latino-Americano de Malformações Congênitas (ECLAMC). Também participa como membro gestor e colaborador do Instituto Nacional de Genética Médica Populacional (INAGEMP).

O método utilizado parte de uma adaptação do enfoque teórico-metodológico DS apoiado num roteiro ampliado para institutos de pesquisa, com ênfase nas ações de ensino e pesquisa e em indicadores mistos de produção, incluindo o modelo aplicado para levantamento da produção científica $^{19}$. Neste estudo foi incorporada a análise relacionada ao apoio e formulação de políticas de saúde, não prevista inicialmente no método, o que constituiu uma adaptação inovadora.

O trabalho envolveu a realização de reuniões/ oficinas com os atores-chave e o levantamento de dados e documentos institucionais no período de dezembro de 2014 a junho de 2015. É importante salientar que o roteiro quanti-qualitativo, baseado nos pressupostos da abordagem DS e adaptado à realidade brasileira, ampliada para institutos de pesquisa ${ }^{19}$, serviu de base para a análise estratégica aqui apresentada e envolveu ampla discussão presencial nas oficinas de trabalho. Todas as notas e todas as fases foram construídas durante as oficinas.

Os atores-chave envolvidos, representados pelo gestor do Centro de Genética Médica, por profissionais, gestores ou responsáveis pelos processos de atenção/setores e profissionais que desenvolvem atividades de ensino, pesquisa, articulação com a rede de serviços e da área de planejamento do IFF, totalizaram 25 participantes, com frequência acima de $90 \%$. A pesquisa foi aprovada pelo CEP/IFF (Parecer no 1.740.977), com o compromisso de divulgar as informações dentro dos preceitos éticos de pesquisa, de acordo com o TCLE assinado pelos participantes.

Foram utilizadas as seguintes etapas do enfoque de gestão estratégica hospitalar (DS) ${ }^{16-18}$ :

1) Análise do existente - composta pelo diagnóstico administrativo e médico que proporciona elementos para a fase de definição dos segmentos de atividades. Foram utilizados os dados dos setores de estatística da unidade, do CGM, do serviço de enfermagem, das vices-diretorias de Pesquisa e Ensino e da base de dados da Plataforma Lattes. 
O diagnóstico administrativo teve como foco a identificação das informações: número de pacientes e perfil da população atendida por categorias, número de exames realizados e levantamento da produção científica. O diagnóstico médico foi baseado na categorização das patologias atendidas, tipo de população, modalidades de atenção de acordo com as especificidades do paciente, tecnologias utilizadas, levantamento das sinergias e parcerias, identificação dos serviços clínicos colaboradores dentro e fora do instituto e tipos de coortes e pesquisas clínicas em andamento. O período considerado para análise dos dados foi o ano de 2014 para os atendimentos clínicos; 2013 e 2014 para os exames laboratoriais e diagnósticos; e 2010 a 2014 para projetos de captação de recursos, artigos científicos publicados e demais produções científicas.

2) Segmentação - refere-se à análise multicritérios e visa o estabelecimento de segmentos homogêneos que permitam uma análise estratégica representativa, considerando a complexidade da missão institucional, sendo que nem sempre todos os grupos de atividades apresentam o mesmo desenvolvimento ou valor estratégico. Neste estudo foram utilizados critérios como: patologias e condição de saúde dos usuários, grau de complexidade, imbricações entre patologias, características do processo de atendimento e linhas de cuidado, tecnologias utilizadas, necessidade de dispor de uma infraestrutura e as competências necessárias.

3) Análise estratégica e da posição competitiva - é uma análise prospectiva e o valor atribuído a cada segmento representa o interesse/prioridade relativo para a especialidade. Os resultados obtidos nesta etapa se referem à hierarquização/ priorização dos segmentos em função de seu valor estratégico, da identificação da problemática específica de cada segmento e da hierarquização dos segmentos em função de sua posição competitiva em termos de excelência. Essa posição é analisada a partir da definição de fatores-chave de sucesso (FCS) pelos participantes, considerando referências nacionais e internacionais, representados por tecnologias, recursos humanos especializados, expertise, capacidades relacionais, capacidades financeiras, entre outros, e o nível de controle pela instituição destes FCS para oferecer com excelência as atividades. Um nível alto de controle dos FCS significa alta competividade.

Utilizou-se para análise de valor dos segmentos os critérios: possibilidades de crescimento, intensidade da concorrência, investimento em equipamentos, investimento em recursos huma- nos, sinergias, possibilidade de parceria externa, potencial de ensino e pesquisa, contribuição para o projeto político geral do instituto, grau de motivação interna, potencial municipal/estadual e potencial regional/nacional. É importante ressaltar que neste estudo todos os critérios foram analisados considerando as quatro funções do instituto: atenção de referência, ensino, pesquisa e apoio à formulação de políticas de saúde.

4) Portfólios de atividade - são representados por gráficos onde são posicionados os segmentos estratégicos de uma especialidade para uma análise de conjunto e valor comparativo. A ordenada representa o valor, a abscissa a posição competitiva. Cada segmento é representado por bolhas, cujos tamanhos correspondem ao volume de produção relativo das atividades, e sua posição depende do escore obtido na avaliação de valor e grau de controle dos FCS, o que define sua posição competitiva. O gráfico é dividido em quatro quadrantes, sendo os dois superiores correspondentes a segmentos de alto valor, os quadrantes à direita a segmentos de alta posição competitiva e os da esquerda a segmentos de baixa competitividade.

Para contemplar a complexidade da missão institucional ${ }^{18}$, envolvendo atividades de pesquisa e ensino, além da assistencial, em instituições onde a pesquisa tem alto valor, foram criados dois portfólios (um referente à produção relativa à atenção à saúde e outro à produção científica) para possibilitar uma visão do conjunto dos segmentos, o diagnóstico do risco estratégico e o volume de produção de forma ampliada ${ }^{19}$.

5) Plano de ação - consiste de um conjunto de ações visando a revalorização de segmentos estratégicos para a organização e para a rede de saúde e a atuação sobre algum critério da análise do valor de baixo escore passível de intervenção (quando a decisão política é buscar aumentar o valor). As ações podem se traduzir em diminuição de atividades quando é mais pertinente ofertá-las na rede de atenção em outras unidades, modificações no modelo assistencial e nas formas de atendimento que caracterizam determinado segmento, ou no perfil de tecnologias utilizadas, incluindo ações de natureza social e intersetorial.

\section{Resultados e discussão}

A partir dos dados obtidos no diagnóstico administrativo e médico, e especialmente considerando os critérios patologia e tecnologia, foram identificados os seguintes segmentos: diagnósti- 
co clínico (anomalias congênitas, doenças degenerativas do adulto, erros inatos do metabolismo, doenças do desenvolvimento, doenças hereditárias e síndromes genéticas); doenças lisossômicas; osteogênese imperfeita (OI); aconselhamento genético (reúne critérios de patologia, tecnologia e linhas de cuidado); citogenética; bioquímica; e genômica.

A determinação do valor prospectivo de cada segmento foi realizada a partir da análise multicritérios (possibilidade de crescimento, concorrência, investimento, sinergia, parceria, potencial de pesquisa e ensino, projeto político, motivação interna, potencial local/estadual e potencial regional/nacional), seguindo orientação do roteiro adaptado para aplicação no Brasil da démarche stratégique ${ }^{16-19}$, permitindo identificar a capacidade da atração do segmento. Os pesos, atribuídos pelos atores participantes das oficinas, encontram-se resumidos na Tabela 1 , juntamente como a nota e escore identificados na análise dos critérios de avaliação para cada segmento.

$\mathrm{Na}$ análise da Tabela 1, observa-se a valorização de todos os segmentos de uma forma relativamente homogênea (escores variando entre 14,23 e 16,21), o que expressa o valor atribuído pelos atores envolvidos a todos os segmentos $\mathrm{e}$ sua importância estratégica para a missão institucional.

A liderança do segmento genômica se deve à evolução tecnológica contemporânea, que ampliou a disponibilidade do diagnóstico de precisão $\mathrm{O}^{21}$, à necessidade de fortalecer o conhecimento médico-clínico nessa área e à priorização desse segmento pelas agências de fomento. Apesar do decréscimo das doenças infantis e a estabilização do indicador epidemiológico, o segmento de diagnóstico clínico também apresenta uma tendência de crescimento, em função da melhora do diagnóstico no pré-natal com maior controle das doenças perinatais e pelo potencial de apoio e assessoria a outras unidades da rede de atenção, visando estimular a criação de novos centros de atendimento.

A projeção de alto crescimento para o segmento de bioquímica se dá pela ampliação do Programa de Triagem Neonatal ${ }^{22}$, pela identificação de novas terapias no campo dos erros inatos do metabolismo, por métodos diagnósticos e biomarcadores mais precisos na atenção à saúde. Em relação ao segmento da citogenética, a tendência de crescimento é justificada pelo fato de ser o único laboratório público que faz diagnóstico citogenético na rede SUS no estado do Rio de Janeiro.

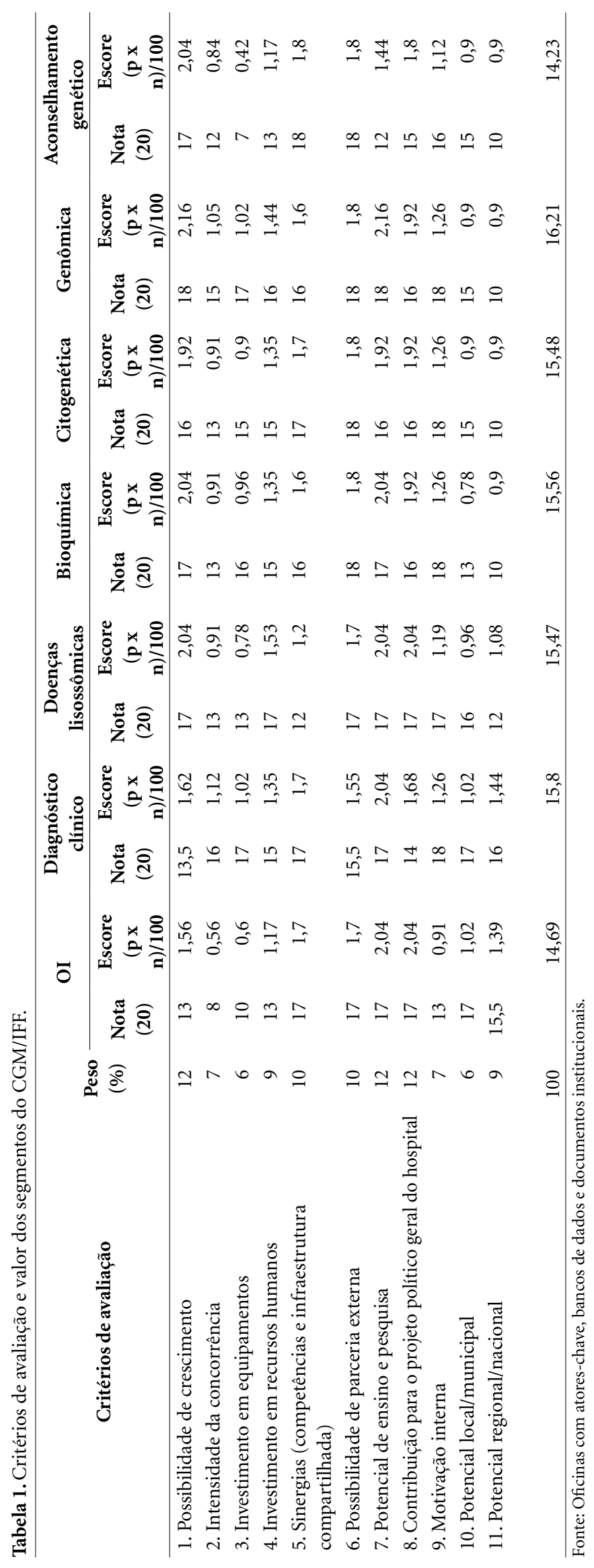


Os segmentos de diagnóstico laboratorial, aconselhamento genético e doenças lisossômicas apresentam, por sua vez, o maior potencial de crescimento. Isso se deve ao fato do IFF ter sido habilitado recentemente como o Centro de Referência para Doenças Raras do Estado do Rio de Janeiro, o que influencia no aumento da demanda por serviços de diagnóstico, prevenção e tratamento ${ }^{9,10}$.

Apesar de todos os segmentos estarem bem posicionados no critério concorrência, os referentes a diagnóstico clínico e genômica apresentaram a melhor posição. A baixa concorrência se deve à pouca oferta desses serviços ${ }^{17}$, e ocorre principalmente por uma resposta ainda insuficiente em termos de políticas, planejamento e investimento para oferta de serviços públicos voltados às enfermidades genéticas ${ }^{5,7}$.

Os segmentos que mais requerem investimentos tecnológicos e equipamentos são os de diagnóstico clínico e laboratórios, em função da evolução tecnológica nessas áreas e do fato de a estrutura atual do Centro de Genética precisar de renovação e incorporação tecnológica para ter um diferencial estratégico maior. Todos os segmentos exigem grande investimento em recursos humanos, considerando-se a insuficiência dos recursos existentes e a tendência de alto crescimento da demanda e à baixa concorrência.

Em relação ao critério sinergia, todos os segmentos obtiveram pontuação alta, devido à interdependência com as diversas especialidades existentes no hospital. No caso das doenças lisossômicas, que obtiveram a menor nota, existe um potencial de aprimoramento da articulação com o ambulatório de pediatria, a enfermagem, os serviços de reabilitação, o laboratório, a farmácia e as coortes de pesquisa do IFF.

Foi identificado também um alto potencial para parcerias em todos os segmentos com centros de referência especializados, clínicas de família, redes de pesquisa (ECLAMC; INAGEMP) e redes de atenção (CROI/IFF; Centros de Doenças Raras), além da necessidade de melhorar os aspectos envolvidos na formalização dessas parcerias. Em relação ao segmento doenças lisossômicas foi apontada a necessidade de desenvolver maior parceria com a atenção primária, para atendimento e viabilização do tratamento pela infusão enzimática no ambiente domiciliar, o que evita deslocamento dos pacientes e também otimiza os recursos hospitalares.

Com exceção do segmento aconselhamento genético, todos os demais apresentam grande atratividade em relação a pesquisa e ensino, bem como para participação em redes de pesquisa ${ }^{23}$. Entre os fatores propulsores da pesquisa, destacam-se: a evolução tecnológica desses segmentos e a sua contribuição ao aprimoramento da investigação diagnóstica (sequenciamento de nova geração de DNA) ${ }^{21}$ e terapêutica (terapia de reposição); o fato de poder realizar articulação com redes de pesquisa nacionais e internacionais; participação em ensaios clínicos de medicamentos de grandes empresas; e a necessidade de definição de diretrizes clínicas nacionais na área. Em relação ao potencial de ensino, foi identificado alto potencial para residência médica (segmento osteogênese imperfeita - OI), cursos de graduação e pós-graduação (segmentos bioquímica e doenças lisossômicas), ampliação das estratégias de formação de profissionais da atenção primária e de outros centros de referência (principalmente em aconselhamento genético e $\mathrm{OI}$ ), o que se mostra como uma estratégia fundamental para a qualificação do cuidado.

Como se trata de uma área especializada e com potencial de realização de ações que correspondem às funções do instituto, todos os segmentos tiveram notas altas no critério de contribuição ao projeto político da organização. A motivação interna das equipes de trabalho também se mostrou elevada e o potencial para atendimento local/estadual e regional/nacional se equiparam na maioria dos segmentos, indicando um alto potencial devido à concentração da oferta de serviços especializados em centros de referência estaduais ${ }^{5}$.

A análise da posição competitiva (etapa 3) se referiu à identificação dos principais fatoreschave de sucesso (FCS) por segmento. Foram utilizadas em torno de seis a nove FCS e o grau de controle foi obtido por meio de uma notação, considerando como parâmetro o grau ideal de controle correspondente a um desempenho de alto nível de qualidade.

Os principais pontos fortes estão representados pelos seguintes FCS: a certificação do CGM como Centro de Referência de Doenças Raras e como Centro de Referência em OI; disponibilização de tecnologia de aconselhamento genético retrospectivo, tecnologia de tratamento de OI e para TRE (terapia de reposição enzimática); expertise profissional e equipamentos básicos disponíveis; sinergia com as áreas de atenção hospitalar e especialidades do IFF; parcerias estabelecidas com outros centros locais e nacionais; e a constituição de coortes de pesquisa e ensaios clínicos. A maioria dos segmentos apresenta bom controle do recurso expertise profissional e dos 
equipamentos básicos necessários à operação dos laboratórios especializados, o que é decisivo para a atuação do CGM/IFF.

$\mathrm{Na}$ análise geral da posição estratégica dos segmentos da genética médica, observou-se que osteogênese imperfeita e doenças lisossômicas apresentam posição competitiva mais alta; os demais segmentos apresentam posição mediana, enquanto bioquímica, genômica e diagnóstico clínico obtiveram as posições mais críticas.

Foi considerada alta a sinergia dos segmentos diagnóstico clínico, OI, doenças lisossômicas e aconselhamento genético, com outros setores e especialidades do IFF e a parceria do segmento OI com o Instituto Nacional de Traumatologia e Ortopedia (INTO) e o Instituto Estadual de Diabetes e Endocrinologia (IEDE), necessária à complementação do tratamento dos pacientes nas especialidades ortopédicas e endocrinológicas. Como o CGM não possui a especialidade bioquímica, é fundamental a parceria com o Laboratório de Erros Inatos do Metabolismo (LABEIM), da Universidade Federal do Rio de Janeiro, para a realização de exames específicos essenciais necessários ao diagnóstico. A possibilidade de fomento à pesquisa, por meio da criação de coortes de pesquisa e ensaios clínicos, é um FCS com alto escore, envolvendo principalmente os segmentos de OI e doenças lisossômicas.

Os FCS com notação abaixo de 12 se referem a pontos mais fracos ou a recursos críticos a serem incorporados, como equipe multiprofissional para os segmentos de diagnóstico clínico e aconselhamento genético. O segmento diagnóstico clínico necessitaria, para fazer frente à complexidade dessa tecnologia e à sua posição como referência, de competências especializadas em diversas áreas profissionais, como neurologia, psicologia, ortopedia, fisioterapia, serviço social e enfermagem. Por outro lado, o segmento aconselhamento genético precisaria de profissionais com formação em psicologia e serviço social, além da incorporação de recursos humanos com expertise em genética clínica, para potencializar sua capacidade de atendimento e ampliar suas ações de pesquisa e ensino ${ }^{23}$.

Quanto aos recursos de infraestrutura, apontou-se a necessidade de aprimoramento das tecnologias de informação (TI) para disponibilização de banco de dados, softwares específicos, suporte especializado e equipamentos para apoiar o crescimento dos segmentos de OI, citogenética e genômica nas funções de atenção e pesquisa. Equipamentos de alta resolução foram identificados como recursos críticos para cito- genética e doenças lisossômicas. $\mathrm{Na}$ área operacional seria necessário reforçar recursos como técnico de laboratório, insumos, estrutura física e manutenção, para apoiar principalmente o segmento de genômica.

$\mathrm{Na}$ etapa 4 deste estudo, os dados de produção relacionados ao portfólio das atividades de atenção à saúde foram obtidos por meio de relatórios internos dos atendimentos realizados pelos segmentos de diagnóstico clínico, doenças lisossômicas, aconselhamento genético e OI, e pelo relatório de exames realizados pelos segmentos de citogenética (interno), bioquímica (externo) e genômica (interno). Na construção do portfólio relacionado à produção científica foram considerados dados de captação de recursos para desenvolvimento dos projetos de pesquisa obtidos por intermédio dos setores internos do hospital. Os dados relacionados à produção de artigos, participação em congressos e cursos e orientações foram obtidos por meio da plataforma Lattes. Os portfólios elaborados foram apresentados em oficina de trabalho, para análise e definição de estratégias para enfrentamento dos pontos fracos.

O portfólio representa o valor e a posição concorrencial dos segmentos estratégicos da genética médica. Sua análise permite uma ideia do conjunto e um diagnóstico do risco estratégico a que o segmento se expõe, resultante da maior ou menor adaptação da oferta de serviços (valor da atividade) e do esforço requerido para se conseguir maior controle sobre os FCS, o que aumenta a posição competitiva e, consequentemente, o atendimento da demanda referente aos segmentos de atividades analisados ${ }^{17-19}$. Permite também sugerir as grandes linhas de ação de acordo com a posição estratégica prospectiva.

Da leitura do portfólio relacionado ao eixo de atenção à saúde (Gráfico 1), podemos observar que todos os segmentos visualizados são de alto valor, indicando uma boa adaptação da genética médica à missão institucional do IFF, representada pela oferta de uma atenção de referência e alta densidade tecnológica. Entre os segmentos de maior atratividade, destacam-se a genômica $(16,21)$, seguida de diagnóstico clínico $(15,8)$, bioquímica $(15,56)$, citogenética $(15,48)$, doenças lisossômicas $(15,47)$, OI $(14,69)$ e aconselhamento genético $(14,23)$. Em relação à posição competitiva, os segmentos apresentam posições diversas, com diagnóstico clínico, bioquímica e genômica em situação de risco estratégico, com escores de 9,79, 9,4 e 10,75, respectivamente. É importante considerar que os desafios para superar essa situação se relacionam também a difi- 


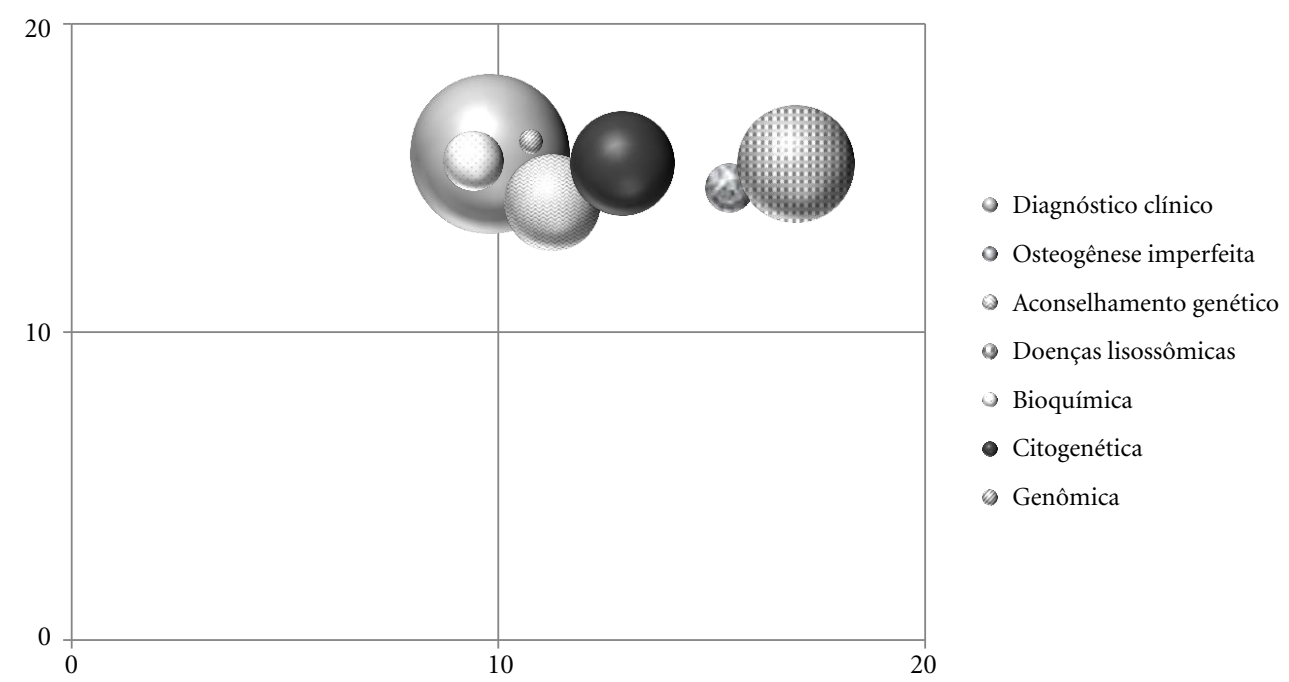

Gráfico 1. Portfólio assistencial.

Fonte: Oficinas com atores-chave, bancos de dados, livros de registros e documentos institucionais.

culdades tanto na regulação da incorporação tecnológica no Brasil $^{24}$ como a questões referentes à viabilidade política e econômica na área da saúde. É fundamental a construção de políticas que possam acelerar a incorporação tecnológica, apoiar pesquisas de ponta envolvendo atores/instituições de diferentes níveis, incluindo os setores público e privado ${ }^{25}$. O direito à saúde garantido em nossa Constituição precisa estar alicerçado em políticas que fortaleçam a democratização da oferta de atenção em todos os níveis, incluindo a especializada e com densidade tecnológica, sem descuidar da articulação com a rede de atenção que o cuidado na área de genética médica exige.

No portfólio correspondente à produção científica (pesquisa e ensino), apresentado no Gráfico 2, observa-se que o segmento diagnóstico clínico é o de maior volume (817,75 pontos), seguido por doenças lisossômicas (385), genômica (236), citogenética (199), osteogênese imperfeita (75), aconselhamento genético $(23,5)$ e bioquímica (16). Para o cálculo da produção científica relacionada a ensino e pesquisa foram utilizados parâmetros com pesos diferenciados, que correspondem a artigos publicados, captação de recursos, participação em congressos, orientações realizadas, participações em bancas de pós-graduação stricto sensu e coordenação de $\operatorname{cursos}^{19}$.

Os portfólios foram apresentados em oficina de trabalho para análise e serviram de base para a definição de estratégias para os diversos segmentos da especialidade, assim como para a elaboração de um plano de ação (etapa 5) para operacionalização das estratégias pactuadas.

As estratégias se basearam nos FCS e pontos fracos identificados. Destacam-se: necessidade de fortalecimento da equipe multiprofissional (neurologista, psicólogo, ortopedista, enfermeiro, fisioterapeuta, assistente social), fundamental para a área de genética, que demanda trabalho interdisciplinar ${ }^{1}$; necessidade de negociação com os coordenadores das áreas técnicas da unidade para aumentar a disponibilidade e o apoio desses profissionais ao CGM; ampliação do quadro técnico, utilizando recursos do orçamento do Centro de Doenças Raras; e estabelecimento de parcerias com a Rede Diagnóstica Genômica da Fiocruz para fins de complementação diagnóstica.

Com relação às demandas de infraestrutura física, as principais necessidades encontram-se no suporte de recursos de tecnologia da informação, insumos e equipamentos estratégicos para área laboratorial e recursos para pesquisa. Seria preciso incorporar ao Plano de Investimentos do Instituto: o desenvolvimento de sistemas operacionais e a aquisição de softwares específicos; planejar a incorporação de insumos e equipamentos; a modernização da estruturação física do laboratório; e o provimento dos serviços de 


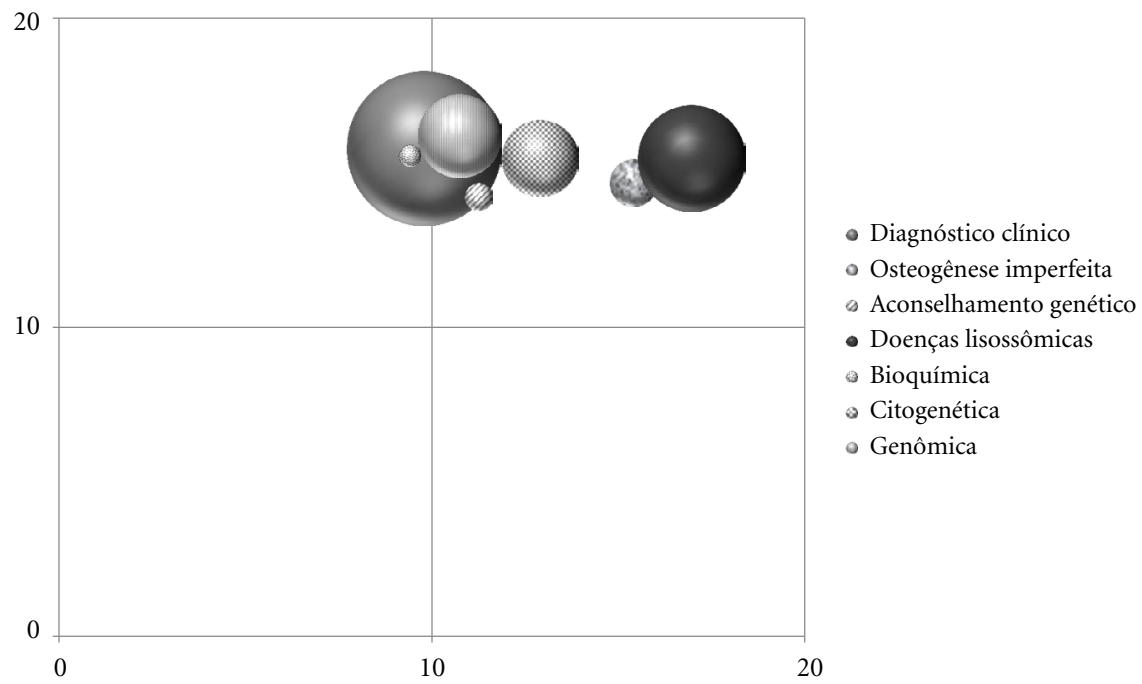

Gráfico 2. Portfólio de produção científica.

Fonte: Oficinas com atores-chave, documentos institucionais e Plataforma Lattes.

manutenção, por meio da plataforma tecnológica compartilhada da Fiocruz.

Estratégias mais específicas foram identificadas por segmento e dizem respeito a agilizar o fluxo de processamento de exames para melhor atender às demandas do segmento diagnóstico clínico, promover ensaios clínicos para o segmento de OI, além de ampliar a capacidade de captação financeira para todos os segmentos laboratoriais e formalizar parcerias com o LABEIM/UFRJ para o segmento de doenças lisossômicas.

É importante problematizar a comparação entre atividades diversas. Assim, utilizamos portfólios diferenciados para atividades de assistência e pesquisa na discussão com os atores nas oficinas de trabalho. O maior ou menor volume de produção, seja de pesquisa ou assistência, não pode ser olhado de forma isolada. O segmento aconselhamento genético, por exemplo, tem alta produção mas está situado na interface dos quadrantes, o que representa um risco estratégico. $\mathrm{O}$ motivo de esse segmento estar situado com escores médios é que a atividade deveria estar mais bem compartilhada com a rede, que foi a estratégia sugerida no plano de ação. A discussão entre o grupo de profissionais e a direção estratégica da instituição contribuiu na tomada de decisões.

O potencial de pesquisa e produção de conhecimento de cada segmento deveria se refletir na produção científica. O posicionamento do segmento citogenética, que apresentou maior volume no portfólio de atenção e menor na produção científica, justifica-se por ser uma atividade diagnóstica. Com a incorporação de novos métodos diagnósticos moleculares ${ }^{21}$ (citogenética molecular pelo CGH Array ou sequenciamento completo do exoma), previstos no plano de atividades, espera-se um aumento na produção científica. A análise que o método propõe é sempre prospectiva ${ }^{16}$.

Há segmentos mais bem posicionados no portfólio assistencial e outros bem situados no portfólio de produção científica. Os motivos que formaram a nota foram considerados para pensar estratégias de reposicionamento dessas atividades. Assim, a baixa produção assistencial em um segmento de alto valor e boa posição estratégica pode indicar a necessidade de mais profissionais com essa expertise, como no exemplo do segmento genômica. Uma alta produção científica em contraposição à menor produção assistencial pode indicar alguns profissionais mais envolvidos em pesquisa/docência e menos nas atividades de assistência. Só uma análise estratégica poderá mostrar a pertinência ou não de se investir na produção assistencial dentro do hospital ou de compartilhar atividades com a rede de cuidados $^{17}$. Em Instituições com missão complexa, a articulação entre assistência, pesquisa e ensino deve ser aprofundada, pois além do atendimento 
à população que demanda serviços, os resultados das pesquisas clínicas articuladas a atividades assistenciais produzem conhecimento que poderá ser incorporado em avanços tecnológicos em diagnóstico ou cuidado, melhores protocolos e na própria formulação de políticas, o que se refletirá em melhoria na saúde e qualidade de vida da população.

\section{Considerações finais}

A partir da política de humanização e da gestão estratégica hospitalar, foram analisados os segmentos alinhados com a nova forma de organização do Instituto Nacional Fernandes Figueira, considerando as sinergias internas com outros setores, o potencial de parcerias externas e a articulação com a rede de cuidados mais abrangente. A análise setorizada não contribui para $\mathrm{o}$ desenvolvimento de proposições mais ousadas ou abrangentes, portanto seguiu-se uma linha prospectiva da especialidade, evitando-se uma visão reduzida.

O estudo mostrou uma boa posição competitiva dos segmentos da especialidade genética, destacando-se a necessidade de investimento em alguns pontos estratégicos, como: equipe interdisciplinar, incorporação de equipamentos médicos e tecnologias de informação, e articulação com a rede de atenção primária e outros centros de referência que compõem essa linha de cuidado.

$\mathrm{O}$ alinhamento à nova missão do IFF traz desafios e a necessidade de desenvolvimento de ações que a área tem capacidade para potencializar: maior articulação com a rede, especialmente com a atenção básica, para ampliar atividades de prevenção por meio do aconselhamento genéti$\mathrm{CO}^{23}$; identificação de estratégias de coordenação e tecnologias de cuidado que permitam desospitalização, cuidado domiciliar e acompanhamento clínico; atenção à demanda e ampliação da oferta, como proposição de linhas de cuidado e centros de referência nacionais para diagnóstico e tratamento; ações e cursos de formação com estratégias de educação a distância para cobertura nacional; e elaboração de diretrizes clínicas e projetos de pesquisa no âmbito da avaliação de incorporação de tecnologias.

Essas estratégias são fundamentais, considerando-se que certas condições genéticas demandam admissão hospitalar precoce, reinternação e cirurgias, e a cronicidade aumenta a carga dos serviços de saúde e os custos da atenção ${ }^{5}$.

As discussões destacaram também que não basta a evolução tecnológica para a solução dos problemas de saúde na área da genética. É preciso avançar na implementação de políticas e no planejamento para ampliar o acesso da população às novas tecnologias, investindo em formação de profissionais de saúde e na articulação dos institutos de pesquisa e serviços de referência com a rede mais ampla de atenção à saúde, bem como na maior articulação pesquisa/assistência/ensino. É necessário, ainda, considerar o cenário macro e fortalecer o papel do Estado por meio de políticas de saúde que articulem as dimensões econômica, social e regulatória e promovam a participação de atores com expertises em diversas áreas do conhecimento capazes de incrementar a cadeia de valor em inovação em saúde ${ }^{25}$.

A metodologia adotada permitiu a criação de um espaço colaborativo e de comunicação que gerou uma discussão estratégica sobre o CGM, com a participação da chefia e de todos os profissionais, acolhendo-se a característica de autonomia das organizações profissionais, a partir de Minstzberg ${ }^{20}$, que exige a participação dos profissionais da ponta, ou centros operadores, na tomada de decisões para que estas se realizem efetivamente. Destaca-se o aprofundamento da análise na área de pesquisa, com a construção de um portfólio específico para a produção científi$\mathrm{ca}^{19}$. Foi incluída a dimensão formulação de políticas, considerando a característica de referência nacional e a contribuição do CGM para a concepção das mesmas, fundamental para indicar o papel diferencial da genética médica no SUS e também para valorizar a construção de propostas no campo da saúde pública. 


\section{Colaboradores}

E Artmann, JC Llerena Junior e LT Pereira trabalharam na concepção, no levantamento e análise das informações, na elaboração e revisão do manuscrito. L Binsfeld e FJU Rivera trabalharam no levantamento e análise das informações, bem como na elaboração e revisão do manuscrito.

\section{Referências}

1. World Health Organization (WHO). World atlas of birth defects. Human Genetics Programme, Management of Non communicable Diseases. International Centre for Birth Defects. WHO: Geneva; 2003.

2. Salzano FM. Saúde pública no primeiro e terceiro mundos: desafios e perspectivas. Cien Saude Colet 2002; 7(1):7-16.

3. Llerena Junior JC. Genética médica, Sistema Único de Saúde brasileiro (SUS) e integralidade na atenção e no cuidado à saúde. Cien Saude Colet 2002; 7(1):17-41.

4. França LB, Lansky S, Rego MAS, Malta DC, França JS, Teixeira R, Porto D, Almeida MF, Souza MFM, Szwarcwald CL, Mooney M, Naghavi M, Vasconcelos AMN. Principais causas da mortalidade na infância no Brasil, em 1990 e 2015: estimativas do estudo de Carga Global de Doença. Rev Bras Epidemiol 2017; 20(Supl. 1):46-60.

5. Horovitz DD, Llerena Juniorr JC, Mattos RA. Atenção aos defeitos congênitos no Brasil: panorama atual. Cad Saude Publica 2005; 21(4):1055-1064.

6. Vieira DKR, Attianezi M, Horovitz DD, Llerena Junior JC. Atenção em genética médica no SUS: a experiência de um município de médio porte. Physis 2013; 23(1):241-261

7. Meira JGC, Acosta AX. Políticas de saúde pública aplicadas à genética médica no Brasil. R. Ci. méd. biol. 2009; 8(2):189-197.

8. Brasil. Portaria GM/MS no 81 , de 20 de janeiro de 2009. Institui, no âmbito do Sistema Único de Saúde (SUS), a Política Nacional de Atenção Integral em Genética Clínica. Diário Oficial da União 2009; 21 jan.

9. Brasil. Portaria GM/MS n ${ }^{\circ} 199$, de 30 de janeiro de 2014. Institui a Política Nacional de Atenção Integral às Pessoas com Doenças Raras, aprova as Diretrizes para Atenção Integral às Pessoas com Doenças Raras no âmbito do Sistema Único de Saúde (SUS) e institui incentivos financeiros de custeio. Diário Oficial da União 2014; 31 jan.

10. Brasil. Portaria GM/MS no 3.123 , de 28 de dezembro de 2016. Habilita o Instituto Nacional Fernandes Figueira (Fiocruz) como Centro de Referência na Atenção Integral às Pessoas com Doenças Raras no âmbito do Sistema Único de Saúde (SUS). Diário Oficial da União 2016; 29 dez.

11. Brasil. Portaria GM/MS n ${ }^{\circ} 4159$, de 21 de dezembro de 2010. Define o Instituto Fernandes Figueira da Fundação Oswaldo Cruz (FIOCRUZ) como Instituto Nacional de Saúde da Mulher, da Criança e do Adolescente Fernandes Figueira da Fiocruz, para atuar como órgão auxiliar do Ministério da Saúde no desenvolvimento, na coordenação e na avaliação das ações integradas para a saúde da mulher, da criança e do adolescente no Brasil. Diário Oficial da União 2010; $22 \mathrm{dez}$.

12. Brasil. Ministério da Saúde. Política Nacional de Humanização: HumanizaSUS [página na Internet]. [acesso 2017 Jul 28]. Disponível em: https://www.gov. br/saude/pt-br/acesso-a-informacao/acoes-e-programas/humanizasus. Brasília; 2013.

13. Santos Filho SB. Pesquisa em humanização: articulações metodológicas com o campo da avaliação. Saude Transform Soc 2014;5(2):1-10. 
14. Artmann E, Rivera FJU. Humanização no atendimento e gestão. In: Deslandes SF, organizadora. Humanização dos cuidados em saúde: conceitos, dilemas e práticas. Rio de Janeiro: Fundação Oswaldo Cruz; 2003. p. 185-218.

15. Crémadez M, Grateau F. Le management stratégique hospitalier. Paris: InterÉditions; 1997.

16. Rivera FJU. A démarche estratégica: a metodologia de gestão do Centro Hospitalar de Lille, França. Cad Saude Publica 1997; 13(1):73-80.

17. Artmann E, Rivera FJU. A démarche stratégique (gestão estratégica hospitalar): um instrumento de coordenação de prática hospitalar baseado nos custos de oportunidade e na solidariedade. Cien Saude Colet 2003; 8(2):479-499.

18. Andrade MAC, Artmann E. Démarche Estratégica em unidade materno-infantil: um instrumento de gestão hospitalar. Rev. Saúde Pública 2009; 43(1):105-114.

19. Artmann E, Andrade MAC, Rivera FJU. Desafios para a discussão de missão institucional complexa: o caso de um Instituto de Pesquisa em Saúde. Cien Saude Colet 2013; 18(1):191-202.

20. Mintzberg H. Estrutura e dinâmica das organizações. Lisboa: Dom Quixote; 2010.

21. Goodwin S, McPherson JD, McCombie R. Coming of age: ten years of next generation sequencing technologies. Nature Reviews Genetics 2016; 17:333-351.

22. Brasil. Ministério da Saúde (MS). Portaria no 822 , de 6 de junho de 2011. Instituir, no âmbito do Sistema Único de Saúde, o Programa Nacional de Triagem Neonatal/PNTN. Diário Oficial da União 2011; 7 jun.

23. Pina Neto JM. Genetic Counseling. J Pediatr 2008; 84(Supl. 4):s20-s26.

24. Krauss-Silva Letícia. Avaliação tecnológica em saúde: questões metodológicas e operacionais. Cad Saude Publica 2004; 20(Supl. 2):s199-s207

25. Gadelha CAG, Temporão JG. Desenvolvimento, inovação e saúde: a perspectiva teórica e política do complexo econômico-industrial da saúde. Cien Saude Colet 2018; 23(6):1891-1902.

Artigo apresentado em 20/04/2019

Aprovado em 30/09/29019

Versão final apresentada em 01/10/2019

Editores-chefes: Romeu Gomes, Antônio Augusto Moura da Silva 